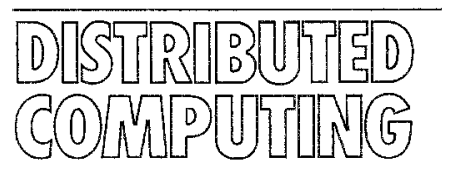

\title{
Efficient detection of a class of stable properties*
}

\author{
Keith Marzullo ${ }^{1}$, Laura S. Sabel ${ }^{2, * *}$ \\ ${ }^{1}$ Department of Computer Science and Engineering, Room 0114, University of California, San Diego, 9500 Gilman Drive, La Jolla, \\ CA 92093-0114, USA \\ ${ }^{2}$ Department of Computer Science, Cornell University
}

Received: April 1992/Accepted: May 1994

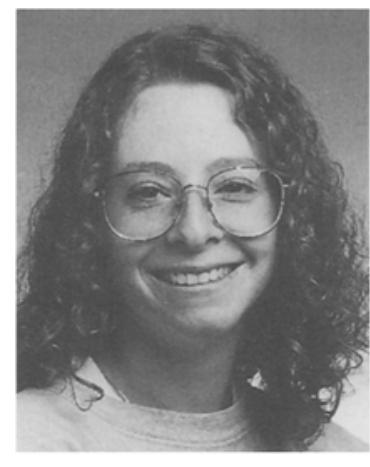

Laura Sabel received the BSE degree from Princeton University in 1989 and the MS degree in Computer Science from Cornell University in 1992. She is currently a $\mathrm{PhD}$ student in the Department of Computer Science at Cornell University. Her research interests include faulttolerance and distributed systems. She is the recipient of an $\mathrm{AT} \& \mathrm{~T} \mathrm{PhD}$ Scholarship.

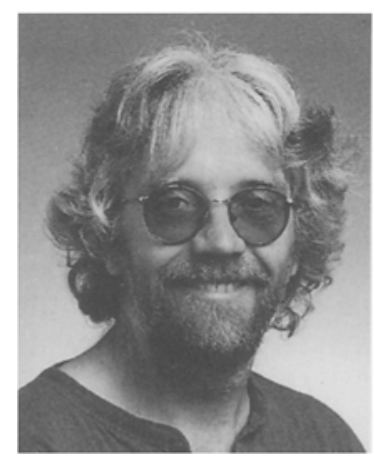

Keith Marzullo received his Ph.D. degree in electrical engineering from Stanford University in 1984 . He is an associate professor in the Computer Science and Engineering Department at the University of California, San Diego. His research interests are in the area of fault-tolerance in both asynchronous and real-time distributed systems. He has consulted on several projects including the IBM Air Traffic Control System, and is an associate editor for IEEE Transactions on Software Engineering.

Summary. We present a general protocol for detecting whether a property holds in a distributed system, where the property is a member of a class of stable properties we call the locally stable properties. Our protocol is based on a decentralized method for constructing a maximal subset of the local states that are mutually consistent, which in turn is based on a weakened version of vector time stamps.

\footnotetext{
* This work was supported by the Defense Advanced Research Projects Agency (DoD) under NASA Ames grant number NAG 2-593, and by grants from IBM and Siemens. The views, opinions, and findings contained in this report are those of the authors and should not be construed as an official Department of Defense position, policy, or decision. An earlier version of this paper appears in the Proceedings of the 5th International Workshop on Distributed Systems, October 1991, Springer-Verlag LNCS Vol. 579

** This author is also supported by an AT\&T PhD Scholarship

Correspondence to: K. Marzullo
}

The structure of our protocol lends itself to refinement, and we demonstrate its utility by deriving some specialized property-detection protocols, including two previouslyknown protocols that are known to be efficient.

Key words: Distributed algorithms - Consistent global states - Distributed snapshots

\section{Introduction}

A stable property is a property that never becomes false once it becomes true. It is conceptually simple to determine whether the global state of a distributed system satisfies a given stable property. One changes the system so that a process uses a snapshot algorithm such as the one presented in [4] in order to collect the relevant local states and channel states and then test to see if the condition holds over the collected state. By repeatedly running a snapshot protocol and testing the collected state, one can eventually detect whether any stable property holds.

Many stable properties are similar in that once the property becomes true, the states of the processes over which the property holds do not change with respect to the property. For example, consider the deadlock property. Once deadlock holds in a system, the states of all of the deadlocked processes are unchanging with respect to deadlock: the processes involved can no longer take any action that might free them from the deadlock or involve them in a new one. Similarly, consider the termination property. Once termination holds, no process can execute an action that could reverse termination. We call such properties locally stable. We will define the class of locally stable properties more formally in Sect. 2.4.

Some locally stable properties, such as deadlock, can be true over a proper subset of the processes in the system. Though the states of the deadlocked processes are stabilized, the same is not true for the rest of the system. Thus, it should be possible to detect deadlock of a set of processes without constructing a complete global state (as is done by taking a global snapshot): a consistent subset comprised of the states of the deadlocked processes is sufficient. It is possible to take advantage of this fact when constructing protocols for detecting deadlock; indeed, the protocol 
given in [6] does this. There are also highly-efficient protocols for detecting termination (e.g., [10]), even though all processes in the system must be stabilized for termination to hold. In general, it is possible to take advantage of the special characteristic of locally stable properties to construct detection protocols that are more efficient than those based on snapshots.

In this paper, we define formally the class of locally stable properties. We also formalize an intuitive variant of vector clocks, and use it to present a new structure known as a consistent subcut. We then present a general method for detecting any locally stable property. Given a system, one first formulates the desired locally-stable property $\varphi$ to be detected. Then, one determines the set of actions that affected processes cannot execute once $\varphi$ holds, and uses this information to derive an efficient detection protocol. The system can then be changed to incorporate this more efficient detection protocol instead of the more expensive global state construction protocols.

We demonstrate our method by using it to derive the protocols of [6] and [10], and then derive an original protocol for detecting a more complicated locally stable property, $k$-out-of- $m$ deadlock.

\section{Definitions}

\subsection{System model}

We consider an asynchronous distributed system consisting of a set of $n$ nonfaulty processes $P=\left\{p_{1}, p_{2}, \ldots, p_{n}\right\}$. The local state of a process consists of the values of the variables of that process. Between any two processes $p_{i}$ and $p_{j}$ there exist two unidirectional nonfaulty FIFO channels: $C_{i, j}$ from $p_{i}$ to $p_{j}$ and $C_{j, i}$ from $p_{j}$ to $p_{i}$. Channels have unbounded delivery time, and processes communicate only by sending and receiving messages over channels. The state of a channel consists of the sequence of messages in transit on that channel.

The execution of a process $p_{i}$ generates a sequence of states $\left(\begin{array}{llll}\sigma_{i}^{0} & \sigma_{i}^{1} & \sigma_{i}^{2} & \ldots\end{array}\right)$ where $\sigma_{i}^{0}$ is the initial state of the process. An event of a process is a pair of adjacent states in this state sequence. We will denote the event that takes process $p_{i}$ from state $\sigma_{i}^{k-1}$ to $\sigma_{i}^{k}$ as $e_{i}^{k}$. When the ordinality of an event or state is not important, we will drop the superscript, e.g. the execution of event $e_{i}$ results in the local state $\sigma_{i}$. Events can be partitioned into send events, receive events, and local events that place a message on a channel, remove a message from a channel, and only change the process's local state respectively. (See [9].)

An arbitrary set $\left\{\sigma_{1}, \ldots, \sigma_{n}, C_{1,2}, \ldots, C_{n, n-1}\right\}$ of local states and channel states may not constitute a sensible global state. A set of local and channel states is called inconsistent if either

- a local state in the set reflects the receipt of a message and no local state in the set reflects the sending of that message, or

- a channel state in the set contains a message and no local state reflects the sending of that message.

A set of local and channel states that is not inconsistent is called consistent [4]. We say that two local states from two processes $p_{i}$ and $p_{j}$ are pairwise inconsistent if the two states cannot be part of the same consistent global state; i.e., the state of process $p_{j}$ reflects the receipt of a message sent by process $p_{i}$ while the state of process $p_{i}$ does not reflect the sending of that message. Note that such a message from $p_{i}$ need not have been received by $p_{j}-$ there need only be a chain of messages between $p_{i}$ and $p_{j}$ such that the end of the chain is reflected in $p_{j}$ 's state and the beginning: of the chain is not reflected in $p_{i}$ 's state. The states of $p_{i}$ and $p_{j}$ are said to be pairwise consistent if they are not pairwise inconsistent. Similarly, we say that two events $e_{i}$ and $e_{j}$ are pairwise consistent iff the two states they produce $\sigma_{i}$ and $\sigma_{j}$ are pairwise consistent.

For notational convenience, we assume for the rest of this paper that channel states are captured in the local states of the processes. There are many ways such a capture could be implemented; a simple way is to have each process maintain a history of all messages that it sends and receives. ${ }^{1}$ If one has such a representation of channel states, then an event is fully defined by a pair of local process states. Furthermore, any collection of local process states, one state per process, is consistent iff each pair of states is pairwise consistent. We will use the term global state to mean a consistent set $\Sigma=\left\{\sigma_{1}, \sigma_{2}, \ldots, \sigma_{n}\right\}$ of the processes' local states.

A consistent cut is defined to be a set of events $C=\left\{e_{1}, e_{2}, \ldots, e_{n}\right\}$ such that the set of states $\left\{\sigma_{1}, \sigma_{2}, \ldots, \sigma_{n}\right\}$ produced by $C$ is a (consistent) global state. Thus, each consistent cut has a corresponding global state. Furthermore, each global state after the initial state $\Sigma^{0}=\left\{\sigma_{1}^{0}, \sigma_{2}^{0}, \ldots, \sigma_{n}^{0}\right\}$ has a corresponding consistent cut: In this sense, global states and consistent cuts are equivalent notions. When defining properties of a distributed system, it is convenient to refer to states; our protocol uses events. For this reason, we define both global states and consistent cuts for use in different contexts.

A property is a set of states of the system. We denote a property with a predicate, expressed over the state of the system, that is true for exactly those states comprising the property. A stable property is a property that continues to be true once it becomes true. ${ }^{2}$. The most often-studied examples of stable properties in distributed systems are deadlock of a subset of the processes, termination of a distributed computation, and the lack of a token among the processes. There are, of course, other stable properties of interest. For example, in a token-passing system that can lose but not generate tokens, the predicate "there are no more than two tokens in the system" is a stable property.

Our method for detecting whether a stable property holds uses the variables that are referenced by the property. The same property, however, may be expressible

\footnotetext{
${ }^{1}$ In an asynchronous system, there is no bound on the number of messages that can be on a channel, and so any representation of a channel's state could require unbounded space. In any real system, however, the number of messages on a channel is bounded, primarily because the amount of buffering available at the sending and receiving processors is limited. For convenience, we will ignore the technical problems that arise from the representation of channel states ${ }^{2}$ Some authors (e.g., [13]) call such a property an invariant
} 
using different variables. For example, consider the property "a subset of the processes are deadlocked" expressed in terms of waits-for graphs [8]. Such a graph contains a node for each process and a directed edge from node $i$ to node $j$ if process $p_{i}$ is waiting for process $p_{j}$. There is some choice involved in determining exactly at what point a process $p_{i}$ is waiting for process $p_{j}$. One can consider "process $p_{i}$ waits for process $p_{j}$ " to hold when $p_{i}$ has sent a message requesting a resource and $p_{j}$ has not sent a message granting that request. One can also consider this to hold when $p_{j}$ has received the request message but not yet responded. If all messages that are sent are eventually received, then both expressions are effective formulations of the property of "waits-for". With our method the exact expression of a property is important. We will refer to such an explicit expression as a formulation $\Phi$ of a property $\varphi$. We will denote with $\sigma_{i} \mid \Phi$ the subset of local state $\sigma_{i}$ that is referenced in the formulation $\Phi$.

Given a formulation $\Phi$, we say that event $e_{i}$ is relevant to formulation $\Phi$ if $e_{i}$ changes the value of a variable referenced in $\Phi$. Since an event is a pair of process states, whether or not an event is relevant can be expressed as a predicate over a pair of states. Thus, the fact that an event is relevant or not is static: it does not change over time.

The definition of an event being relevant depends on the formulation of the property, which in turn depends on the variables used in the formulation. Clearly, one can have formulations that unnecessarily reference variables, thereby making events unnecessarily relevant. For example, suppose that $\Phi$ references variables $x, y, u, v$, but in all states that satisfy $\Phi, x=f(u, v)$ for some function $f$. Then, either the variables $u, v$ are unnecessary or the variable $x$ is unnecessary. In essence, unnecessary variables do not discriminate between a state that satisfies $\Phi$ and one that does not. More precisely, suppose $\Phi$ references variables $x$ and $y$. If the state $\sigma$ satisfies $\Phi$, yet for all states $\sigma^{\prime}$ that have the same value of $y$ but different values of $x, \sigma^{\prime}$ satisfies $\Phi$, then the variable $x$ is unnecessary in $\Phi$. We will assume that the formulations do not reference unnecessary variables.

In order to be able to detect properties without being limited by the exact variables that were used in writing a program, we allow a formulation to reference "variables" that are defined to be local state functions - that is, functions of the variables of a process. Then, an event can be relevant because it changes the value of such a function. For example, consider a program that runs $n \geqq 2$ identical copies of the following process:

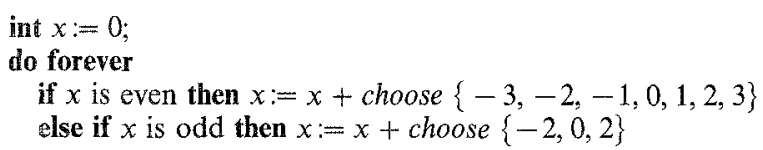

In this program, choose is a function that fairly returns one of the values of the set, and never chooses a value that will cause the addition to $x$ to generate an out-of-range value. Let $x_{i}$ represent process $p_{i}$ 's value of $x$. The property " $\exists i, j: i \neq j: x_{i}$ and $x_{j}$ are odd" is a stable property of this program. This property refers to the parity of $x_{i}$, which can be represented by the state function odd $x_{i}$ that is true if $x_{i}$ is odd and false if $x_{i}$ is even. The property then becomes $\exists i, j: i \neq j: o d d_{-} x_{i} \wedge$ odd $\alpha_{-} x_{j}$. And so, the relevant events of

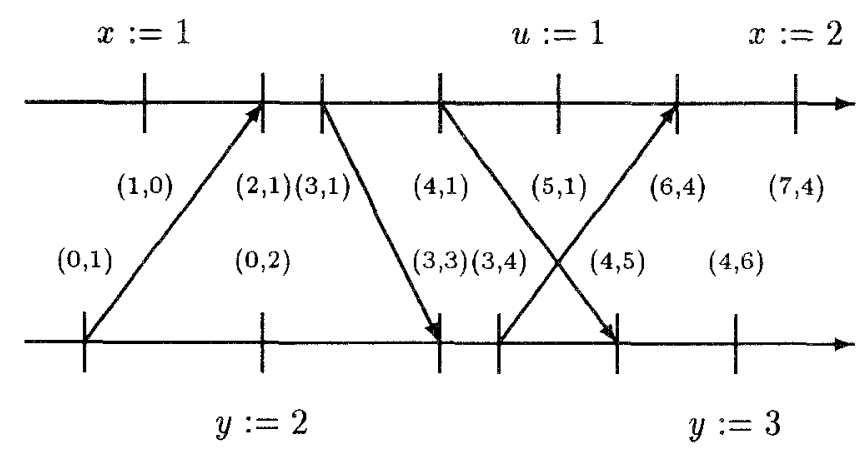

Fig. 1. Execution with vector clocks

process $p_{i}$ with respect to this formulation are those that change the parity of some $x_{i}$.

\subsection{Vector clocks}

Our protocol is based on a variant of vector clocks ([11]) using Lamport's notion of causality (the happened-before relation of [9]). Lamport defines the happened-before relation as follows:

Given two events $e_{i}$ and $e_{j}, e_{i} \rightarrow e_{j}$ iff:

1. $i=j$ and $e_{i}$ is executed before $e_{j}$; or

2. $i \neq j, e_{i}$ is the sending of a message, and $e_{j}$ is the receipt of that message; or

3. there exists an event $e_{k}$ such that $e_{i} \rightarrow e_{k}$ and $e_{k} \rightarrow e_{j}$.

We say that $e_{i}$ happens before $e_{j}$ if and only if $e_{i} \rightarrow e_{j}$.

In the usual definition of a vector clock $V([11])$, each event $e_{i}$ has an $n$-component vector $V\left(e_{i}\right)[1 \ldots n]$ associated with it. $V\left(e_{i}\right)$ is called the vector timestamp of $e_{i}$. The components of $V\left(e_{i}\right)$ are:

- $V\left(e_{i}^{t}\right)[i]=t$; that is, $V\left(e_{i}^{t}\right)[i]$ is the number of events that $p_{i}$ has executed up to and including $e_{i}^{t}$.

- $V\left(e_{i}^{t}\right)[j], j \neq i$ is the number of events $p_{j}$ has executed that happen before $e_{i}^{t}$.

As an example, Fig. 1 shows a space-time diagram of a two-process system with the events labeled by vector clocks.

A simple implementation of vector clocks has process $p_{i}$ maintain an $n$-element vector $V_{i}$ of counters. Process $p_{i}$ increments $V_{i}[i]$ whenever it executes an event $e_{i}$. If $e_{i}$ is a local event or a send event, then $V\left(e_{i}\right)=V_{i}$. If $e_{i}$ is a send event, then $p_{i}$ includes $V\left(e_{i}\right)$ in the message. If $e_{j}$ is the corresponding receive event, then process $p_{j}$ sets $\forall k: k \neq j: V_{j}[k]$ to the maximum of the previous value of $V_{j}[k]$ and the value of $V_{i}[k]$ in the message, and then $V\left(e_{j}\right)=V_{j}$.

Vector timestamps can be used to determine whether two events are related by the happens-before relation. From the definition of vector clocks, one can easily derive the following relationship:

$\forall i, j: i \neq j: V\left(e_{i}\right)[i] \leqq V\left(e_{j}\right)[i] \equiv e_{i} \rightarrow e_{j}$

Furthermore, we can use vector clocks to determine whether two events are pairwise consistent. Two events $e_{i}^{t}$ and $e_{j}^{u}$ are pairwise inconsistent only if (without loss of generality) $e_{i}^{t} \rightarrow e_{j}^{u} \wedge \exists e_{i}^{t^{\prime}}: e_{i}^{t} \rightarrow e_{i}^{t^{\prime}} \rightarrow e_{j}^{u}$, or in terms of 


$$
\begin{aligned}
& \forall i, j: i \neq j: V\left(e_{i}\right)[i] \leqq V\left(e_{j}\right)[i] \equiv e_{i} \rightarrow e_{j} \\
& \left(V\left(e_{i}\right)[i] \geqq V\left(e_{j}\right)[i]\right) \wedge \\
& \quad\left(V\left(e_{j}\right)[j] \geqq V\left(e_{i}\right)[j]\right) \equiv e_{i} \text { and } e_{j} \text { are pairwise consistent } \\
& \forall i, j: V\left(e_{i}\right)[i] \geqq V\left(e_{j}\right)[i] \equiv\left\{e_{1}, \ldots, e_{n}\right\} \text { is a consistent cut }
\end{aligned}
$$

Fig. 2. Equations relating vector timestamps to happens-before and consistency

vector clocks, $V\left(e_{i}^{t}\right)[i]<V\left(e_{j}^{u}\right)[i]$. This gives us the following equation:

$$
\begin{aligned}
\left(V\left(e_{i}\right)[i]\right. & \left.\geqq V\left(e_{j}\right)[i]\right) \wedge \\
\left(V\left(e_{j}\right)[j]\right. & \left.\geqq V\left(e_{i}\right)[j]\right) \\
& \equiv e_{i} \text { and } e_{j} \text { are pairwise consistent }
\end{aligned}
$$

A cut $C=\left\{e_{1}, \ldots, e_{n}\right\}$ is a consistent cut if and only if every pair of events in $C$ is pairwise consistent. Thus, from Eq. 2,

$$
\forall i, j: V\left(e_{i}\right)[i] \geqq V\left(e_{j}\right)[i] \equiv\left\{e_{1}, \ldots, e_{n}\right\}
$$

is a consistent cut

Equations 1, 2, and 3 are summarized in Fig. $2 .^{3}$

\subsection{Weak vector clocks}

In practice, not all events are of interest to applications using vector clocks, and as a result only "important" events are counted by vector clocks while unimportant events are not. For example, some causal broadcast protocols are based on vector clocks that are updated only at send or broadcast events; other events (i.e., receive and local events) do not increment the local component of $V[14,2]$.

By choosing which events update the local component of $V$, one is really choosing an appropriate level of abstraction. In many cases, Eqs. 1-3 continue to hold for the level of abstraction chosen. However, if not all send events cause vector clocks to be updated, then Eqs. 2 and 3 need not hold. For example, suppose $e_{i}^{i}$ and $e_{j}^{u}$ are pairwise inconsistent: $\left(e_{i}^{t} \rightarrow e_{j}^{u}\right) \wedge\left(\exists e_{i}^{t^{\prime}}: e_{i}^{t} \rightarrow e_{i}^{t^{t}} \rightarrow e_{j}^{u}\right)$. If the local component of the vector clock is not updated for all such events $e_{i}^{t^{\prime}}$, then $V\left(e_{i}^{t}\right)[i]=V\left(e_{j}^{u}\right)[i]$ and $V\left(e_{j}^{u}\right)[j]>$ $V\left(e_{i}^{t}\right)[j]$. This satisfies the left side of Eq. 2 implying that $e_{i}^{t}$ and $e_{j}^{u}$ are pairwise consistent, which contradicts our original assumption. It is also possible for $V\left(e_{i}^{t}\right)$ to equal $V\left(e_{j}^{u}\right)$ for some two distinct events $e_{i}^{t}$ and $e_{j}^{u}$. Equation 1 then implies that both $e_{i}^{t} \rightarrow e_{j}^{u}$ and $e_{j}^{u} \rightarrow e_{i}^{t}$, which is impossible for any two events.

For our purposes, only the execution of a relevant event causes the local counter of a vector clock to be updated. This is because the execution of a nonrelevant event does not change the state of the system with respect to $\Phi$. Yet, not all send events need be relevant events, and so the resulting clocks need not satisfy Eqs. 1-3. However, this in fact poses no real problem. Continuing the example given

\footnotetext{
${ }^{3}$ Further information on vector clocks and their properties can be found in surveys on vector clocks, e.g., [16] and [1]
}

in the last paragraph, if $e_{i}^{z^{\prime}}$ does not result in $p_{i}$ 's vector clock being updated, then $e_{i}^{t^{\prime}}$ is not relevant. Hence, $\sigma_{i}^{t}\left|\Phi=\sigma_{i}^{t^{\prime}}\right| \Phi$. And, since there exists an event $e_{i}^{t^{\prime}}$ that is pairwise consistent with $e_{j}^{u}, e_{i}^{t}$ and $e_{j}^{u}$ are pairwise consistent with respect to $\Phi$.

Since our vector clocks may not satisfy Eqs. 1-3, we will call them weak vector clocks. In particular, we denote with $V_{\Phi}$ the weak vector clock for property $\Phi$, which is the vector clock in which $V_{\Phi}\left(e_{i}\right)[i]$ counts only the events relevant to $\Phi$ that $p_{i}$ has executed through $e_{i}$. The weak vector timestamp associated with several events of $p_{i}$ may have the same value, but all such events result in the same local state relative to $\Phi$. For example, in the case of deadlock the relevant events are sending a request for a resource, sending a grant of a resource, and receiving a grant of a resource (see Sect. 4.3). If a process $p$ requests a resource and subsequently sends an unrelated message, then the send event does not change the local state of $p$ with respect to possible deadlock. So, the send event is given the same weak vector timestamp as the resource request event.

Equations 1,2, and 3 can be modified so that they hold for weak vector timestamps. Define an equivalence relation $\sim_{\Phi}$ where two events $e_{i}^{t}$ and $e_{i}^{t^{\prime}}$ are equivalent with respect to $\Phi$, written $e_{i}^{t} \sim_{\phi} e_{i}^{t^{\prime}}$, if $e_{i}^{t}$ and $e_{i}^{t^{\prime}}$ have the same weak vector timestamp. Note that if $e_{i}^{t} \sim_{\Phi} e_{i}^{t^{r}}$ then $\sigma_{i}^{t}\left|\Phi=\sigma_{i}^{t^{\prime}}\right| \Phi$. Similarly, two consistent cuts $C=$ $\left\{e_{1}^{t_{2}}, \ldots, e_{n}^{t_{n}}\right\}$ and $C^{\prime}=\left\{e_{1}^{t_{1}^{\prime}}, \ldots, e_{n}^{t_{n}^{\prime}}\right\}$ are equivalent with respect to $\Phi$, written $C \sim \sim_{\phi} C^{\prime}$, if for all $i, e_{i}^{t_{i}} \sim_{\phi} e_{i}^{t_{i}}$.

Weak vector clocks can be used to determine if two equivalence classes of events are related by the happensbefore relation:

$$
\begin{aligned}
\forall i, j: i \neq j: V_{\Phi}\left(e_{i}^{t}\right)[i] \leqq V_{\Phi}\left(e_{j}^{u}\right)[i] \equiv & \exists e_{i}^{t^{\prime}}, e_{j}^{u^{\prime}}: \\
& \left(e_{i}^{e^{\prime}} \sim{ }_{\Phi} e_{i}^{t}\right) \wedge\left(e_{j}^{u^{\prime}} \sim{ }_{\phi} e_{j}^{u}\right): \\
& e_{i}^{t^{\prime}} \rightarrow e_{j}^{u^{\prime}}
\end{aligned}
$$

Weak vector timestamps can also be used to determine whether two equivalence classes are pairwise consistent:

$$
\begin{gathered}
\left(V_{\Phi}\left(e_{i}^{t}\right)[i] \geqq V_{\Phi}\left(e_{j}^{u}\right)[i]\right) \wedge \\
\left(V_{\Phi}\left(e_{j}^{u}\right)[j] \geqq V_{\Phi}\left(e_{i}^{t}\right)[j]\right) \equiv \exists e_{i}^{t^{\prime}}, e_{j}^{u^{\prime}}:\left(e_{i}^{t^{\prime}} \sim_{\Phi} e_{i}^{t}\right) \wedge\left(e_{j}^{u^{\prime}} \sim_{\Phi} e_{j}^{u}\right): \\
\quad e_{i}^{t^{\prime}} \text { and } e_{j}^{u^{\prime}} \text { are pairwise consistent }
\end{gathered}
$$

Finally, weak vector timestamps indicate whether an equivalence class of cuts is consistent:

$$
\begin{aligned}
\forall i, j: V_{\Phi}\left(e_{i}\right)[i] \geqq V_{\Phi}\left(e_{j}\right)[i] \equiv & \exists \text { consistent cut } C^{\prime}: \\
& C^{\prime} \sim_{\Phi}\left\{e_{1}, \ldots, e_{n}\right\}
\end{aligned}
$$

Equations 4, 5, and 6 are summarized in Fig. 3.

In order to illustrate weak vector clocks, Fig. 4 shows weak vector clock values for the execution shown in Fig. 1, where we assume that the formulation of the property of interest references $x$ and $y$, but not $u$ nor any of the channel states. Note that although the events $x:=1$ and $y:=3$ do not form a consistent cut, their timestamps satisfy Eq. 6 since there exist several consistent cuts equivalent to this inconsistent cut with respect to $\Phi$ (all necessarily having $\langle x=1, y=3\rangle)$. Hence, the two events are consistent with respect to $\Phi$. 


$$
\begin{aligned}
& \forall i, j: i \neq j: V_{\Phi}\left(e_{i}^{t}\right)[i] \leqq V_{\Phi}\left(e_{j}^{u}\right)[i] \equiv \exists e_{i}^{t^{\prime}}, e_{j}^{u^{\prime}}:\left(e_{i}^{t^{\prime}} \sim{ }_{\Phi} e_{i}^{t}\right) \wedge\left(e_{j}^{u^{\prime}} \sim_{\Phi} e_{j}^{u}\right): \\
& e_{i}^{t^{\prime}} \rightarrow e_{j}^{u^{\prime}} \\
&\left(V_{\Phi}\left(e_{i}^{t}\right)[i] \geqq V_{\Phi}\left(e_{j}^{u}\right)[i]\right) \wedge \\
&\left(V_{\Phi}\left(e_{j}^{u}\right)[j] \geqq V_{\Phi}\left(e_{i}^{t}\right)[j]\right) \equiv \exists e_{i}^{t^{\prime}}, e_{j}^{u^{\prime}}:\left(e_{i}^{t^{\prime}} \sim_{\Phi} e_{i}^{t}\right) \wedge\left(e_{j}^{u^{\prime}} \sim_{\Phi} e_{j}^{u}\right): \\
& e_{i}^{t^{\prime}} \text { and } e_{j}^{u^{\prime}} \text { are pairwise consistent } \\
& \forall i, j: V_{\Phi}\left(e_{i}\right)[i] \geqq V_{\Phi}\left(e_{j}\right)[i] \equiv \exists \text { consistent cut } C^{\prime}: \\
& C^{\prime} \sim_{\Phi}\left\{e_{1}, \ldots, e_{n}\right\}
\end{aligned}
$$

Fig. 3. Equations relating weak vector timestamps to happens-before and consistency

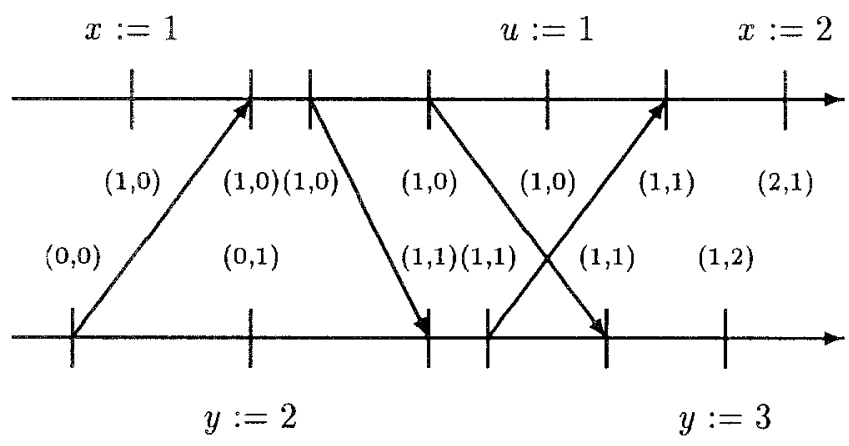

Fig. 4. Execution with weak vector clocks

\subsection{Locally stable properties}

Our protocol will detect a subset of the stable properties that we call locally stable properties. Informally, a stable property $\varphi$ is locally stable if no process involved in the property can change its state relative to $\varphi$ once $\varphi$ holds. For example, suppose $\varphi$ is "processes $p_{i}$ and $p_{j}$ are deadlocked." The property $\varphi$ is locally stable because once $\varphi$ becomes true, neither $p_{i}$ nor $p_{j}$, the processes involved in $\varphi$, can execute any event that could affect $\varphi$ (e.g., requesting or granting a resource). Hence, for any formulation $\Phi$ of $\varphi, \sigma_{i} \mid \Phi$ and $\sigma_{j} \mid \Phi$ remain constant once $\varphi$ holds.

More formally, let $\mathscr{G}$ be the set of all global states that the system can attain. For any $\Sigma \in \mathscr{G}$, define $\Sigma \mid \Phi$ to be the subset of $\Sigma$ that is referenced in the formulation $\Phi$ of $\varphi$, and given a set of processes $Q$ define $\Sigma_{Q}$ to be the subset of $\Sigma$ that consists of the states of the processes in $Q$ (and, by our representation of channel states, the states of the channels between processes in $Q$ ). Finally, define $A_{\Phi}(\Sigma)$ to be the set of processes $p_{i} \in P$ such that $\sigma_{i} \mid \Phi$ does not change in any execution starting in state $\Sigma$.

Definition 1. $\varphi$ is locally stable if and only if there exists a formulation $\Phi$ of $\varphi$ such that for any $\Sigma \in \mathscr{G}$ that satisfies $\Phi$ and for all $\Sigma^{\prime} \in \mathscr{G}$ where $\Sigma_{A_{\phi^{(\Sigma)}}}^{\prime}\left|\Phi=\Sigma_{A_{\Phi^{(\Sigma)}}}\right| \Phi, \Sigma^{\prime}$ satisfies $\Phi$.

In other words, $\varphi$ can be determined from only the states of the processes in $A_{\Phi}(\Sigma)$. Note that $A_{\Phi}(\Sigma)$ can be empty, but only for trivial properties; if $A_{\Phi}(\Sigma)$ is empty, then $\varphi$ can be determined without knowledge of the state of any process or channel, and must therefore be valid in all states in $\mathscr{G}$. For this reason, we will assume in this paper that $A_{\Phi}(\Sigma)$ is nonempty.
The commonly-studied stable properties of deadlock, termination, and lack of a token are all locally stable. For example, if $\Sigma$ is a deadlock state, then $A_{\Phi}(\Sigma)$ includes the processes that are deadlocked in $\Sigma$, and so the presence of deadlock can be determined by considering only the states of the processes in $A_{\Phi}(\Sigma)$. An example of a stable property that is not locally stable is the property "there are no more than $k: k>0$ tokens" in a system where tokens cannot be created but can be lost when passed. This is because if $\Sigma$ is a state in which there are $k$ tokens, then every process can execute a relevant event (namely, it can pass tokens), thereby changing its local state relative to $\Phi$ an unbounded number of times, and so $A_{\Phi}(\Sigma)$ is empty. The property, however, is not valid in all states of the token passing system, so it is not locally stable. $^{4}$

\section{Protocol}

\subsection{Basic protocol}

We first assume that a process $p_{0}$ will determine whether the global state of the processes $P=\left\{p_{1}, \ldots, p_{n}\right\}$ satisfies a locally stable property $\varphi$, given formulation $\Phi$. In Sect. 3.2 , we change this protocol so that any number of processes in $P$ may concurrently assume the role of $p_{0}$.

Our protocol is based on the notion of a consistent subcut - a set of events whose timestamps satisfy Eq. 6 . Informally, the protocol is as follows. Whenever a process $p_{i}$ executes a relevant event $e_{i}^{t}$ (that is, whenever there is a difference between $\sigma_{i}^{t-1} \mid \Phi$ and $\left.\sigma_{i}^{t} \mid \Phi\right), p_{i}$ records in a buffer $B_{i}$ the values $\sigma_{i}^{t} \mid \Phi$ and the vector time stamp $V_{\Phi}\left(e_{i}^{t}\right)$ as $B_{i} . \sigma$ and $B_{i} . V$, respectively. Process $p_{0}$ periodically collects the values of the buffers in any order, yielding a set $\mathscr{B}=\left\{B_{1}, B_{2}, \ldots, B_{n}\right\}$. Once $p_{0}$ has constructed this set, $p_{0}$ determines if there exists a subset of $\mathscr{B}$ that represents a consistent subcut and that satisfies $\Phi$. If $p_{0}$ can find such a subset, then $\Phi$ must currently hold. Note that $p_{0}$ need not examine all consistent subcuts; if $A^{\prime} \subseteq A$ and $\Phi$ holds in $\Sigma_{A^{\prime}} \mid \Phi$, then $\Phi$ will also hold in $\Sigma_{A} \mid \Phi$. So, we need examine only the consistent subcuts of $\mathscr{B}$ that are maximal in size. Of course, each subcut must be large enough for $p_{0}$ to be able to determine whether $\Phi$ holds; for example, $\Phi$ may be of the form $\forall p_{i}: \Psi\left(p_{i}\right)$, in which case only a full consistent cut will satisfy $\Phi$.

Unfortunately, given an arbitrary $\mathscr{B}$, the number of subcuts maximal in size is $\Omega\left(2^{n}\right)$. Fortunately, it is not necessary for $p_{0}$ to examine all of these subcuts. Suppose that the first global state in which $\varphi$ holds is $\Sigma^{f}$ and that the buffers all record local states that are either at or after $\Sigma^{f}$. Further suppose that the two states $B_{i} . \sigma$ and $B_{j}, \sigma$ are not

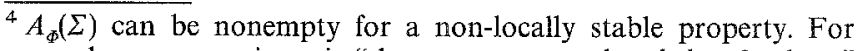
example, suppose $\varphi$ is again "there are no more than $k: k>0$ tokens" and the token passing system consists of red and green processors. Furthermore, only red processors can lose tokens and a green processor never passes a token to a red processor. In this system and for all $\Sigma, A_{\Phi}(\Sigma)$ is the set of green processors (green processors never execute a relevant event), yet the validity of $\varphi$ depends on the states of both the green and red processors. Hence, $\varphi$ is not locally stable
} 
- Each process $p_{i} \in P$ has an associated buffer $B_{i}=(\sigma, V)$ that is initially $\left(\sigma_{i}^{0},[0,0, \ldots, 0]\right)$.

- Whenever process $p_{i}$ executes a relevant event $e_{i}^{t}, p_{i}$ sets $B_{i}$ to $\left(\sigma_{i}^{t}, V\left(e_{i}^{t}\right)\right)$.

- Periodically, $p_{0}$ collects all of the buffers $B_{i}$ and extracts from them the latest subcut $\left\{\sigma_{i}: \forall j: B_{j}, V[i] \leqq B_{i}, V[i]\right\}$.

- $p_{0}$ detects $\Phi$ if $\Phi$ holds on the latest subcut.

Fig. 5. Basic protocol

pairwise consistent in that $B_{i}, V[i]<B_{j}, V[i]$. Since these two states violate Eq. 5, they cannot both be part of the same consistent subcut. However, $B_{i} . V[i]<B_{j} . V[i]$ implies that $p_{i}$ executed a relevant event after global state $\Sigma^{f}$, and so $p_{i} \notin A_{\Phi}\left(\Sigma^{f}\right)$. From the definition of locally stable, the formulation $\Phi$ is determinable from only the states in $A_{\Phi}\left(\Sigma^{f}\right)$, and so $p_{0}$ need not consider subcuts containing $B_{i}, \sigma$.

Thus, given a set of buffered values $\mathscr{B}$ and the partial order $>$, where $\left(\forall B_{i}, B_{j} \in \mathscr{B}: B_{i}>B_{j} \stackrel{\text { def }}{=} B_{i} . V[j]>B_{j} . V[j]\right)$, $p_{0}$ need only find the greatest elements of $\mathscr{B}$ with respect to $\succ$, which can be done in $\Omega\left(n^{2}\right)$ time. ${ }^{5}$ We call this subcut the latest subcut of $\mathscr{B}$. The latest subcut is clearly maximal in size, since all states that are not part of the latest subcut are not consistent with some state in the latest subcut. This gives us the protocol shown in Fig. 5 .

The soundness of this protocol is straightforward - the latest subcut is a consistent subcut, and since $\Phi$ is stable, if $\Phi$ holds in the latest subcut then $\Phi$ currently holds. We now argue that the protocol is complete as well; that is, if $\Phi$ holds, then our protocol will detect $\Phi$. Let $\Sigma^{f}$ be the first global state in which $\Phi$ holds, and let $\sigma_{t}^{f}$ be the local state of process $p_{i}$ in $\Sigma^{f}$. Since $\Phi$ is locally stable, all events that a process $p_{i} \in A_{\phi}\left(\Sigma^{f}\right)$ executes after $e_{i}^{f}$ are not relevant, and so once $B_{i}$ contains $\sigma f$ it will not be changed further. Hence, $p_{0}$ will eventually collect the states $\Sigma_{A_{\Phi}\left(\Sigma^{f}\right)} \mid \Phi$, and since $\Phi$ is locally stable, $p_{0}$ will detect $\Phi$.

\subsection{Decentralization}

In the above protocol, $p_{0}$ 's role is to collect the local states, determine the latest subcut, and check if $\Phi$ holds in this subcut. We can decentralize these steps by collecting the local states in a token.

Consider a token $K$ that consists of $n$ entries $\left\langle D_{1}, \ldots, D_{n}\right\rangle$ where each entry $D_{i}=\left(B_{i}, \sigma, B_{i}, V[i]\right)$; that is, $D_{i}$ will hold the state of $p_{i}$ relevant to $\Phi$ and the local component of $p_{i}$ 's vector clock when it generated that state. Assume that there exists a special value $\perp$ for $D_{i}$ indicating that the state is not in the token; all of the $D_{i}$ in $K$ are initially set to $\perp$.

To determine whether $\Phi$ holds, a process generates a token $K$, inserts its state and local vector clock value into $K$, and passes the token to any other process.

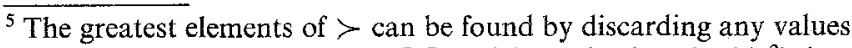
$B_{j}$ such that $\exists i: B_{i}, V[j]>B_{j}, V[j]$, which can be done in $O\left(n^{2}\right)$ time using a straightforward algorithm. And, if all values are incomparable then all the values are greatest elements of $\succ$. To determine that they are all incomparable takes $n^{2}$ comparisons, and so the problem is $\Omega\left(n^{2}\right)$
}

- Each process $p_{i} \in P$ recorás $\sigma_{i}$ and $V_{\phi}\left(e_{i}\right)$ in buffer $B_{i}$ upon executing a relevant event $e_{i}$.

- When $p_{i}$ wants to detect $\Phi, p_{i}$ generates a token $\left\langle D_{1}:=\perp, \ldots, D_{n}:=\perp\right\rangle$, sets $D_{i}$ to $\left(B_{i}, \sigma, B_{i}, V[i]\right)$, and forwards the token to any other process.

When $p_{j}$ receives a token:

- $p_{j}$ sets $D_{j}$ to $\left(B_{j}, \sigma, B_{j}, V[j]\right)$.

- For each $D_{k}: k \neq j, D_{k} \neq \perp, p_{j}$ sets $D_{k}:=\perp$ if $D_{k} \cdot V=B_{k} \cdot V[k]<$ $B_{j}, V[k]$.

$-p_{j}$ determines if $\Phi$ holds on the state values in the token. If not, $p_{j}$ forwards the token to any $p_{k}$ such that $D_{k}=1$.

Fig. 6. Decentralized protocol

When a process $p_{j}$ receives a token $K$, it takes the following steps:

1. Set $D_{j}$ to $\left(B_{j}, \sigma, B_{j}, V[j]\right)$.

2. For all non- $\perp$ values of $D_{k}$ that are not in the latest subcut, set $D_{k}$ to $\perp$.

3. Determine whether the state values in $K$ satisfy $\Phi$. If so, then the detection is made; otherwise, $p_{j}$ forwards the token to a process $p_{k}$, chosen fairly, that has $D_{k}=\perp$. If there is no such process, then $p_{j}$ can either drop the token or, when $p_{j}$ computes a new value of $B_{j}, p_{j}$ can restart at Step 1 with this token.

Note that when process $p_{j}$ executes Step 2, $B_{j}$ must be part of the latest subcut. If it were not, then there would exist a recorded value of $B_{\ell}$ in $D_{\ell}$ such that $B_{j} . V[j]<B_{\ell} . V[j]$. This would imply that $p_{\ell}$ knows of a relevant event executed by $p_{j}$ that resulted in a state that happens after the state recorded in $B_{j}$, which would violate the definition of $B_{j}$. Thus, only the earlier values $D_{k}$ need be tested with respect to $B_{j}$. From above, the value $B_{k}$ in $D_{k}$ can be discarded if $B_{k} . V[k]<B_{j} . V[k]$. The value $B_{k} . V[k]$ is stored in $D_{k} . V$, and so the token $K$ carries enough information for $p_{j}$ to make this test.

The resulting protocol is summarized in Fig. 6. Note that we have no a priori restriction on how many tokens there can be in the system at any time or on the order in which the token is passed, other than that it is passed in a fair manner. These decisions can be made when the protocol is applied to a particular problem. If the protocol is initiated in a state in which $\Phi$ holds, then $\Phi$ will be detected with no more than $n$ token passes.

This protocol is both sound and complete: if $\Phi$ holds when the protocol is initiated; then $\Phi$ will be detected eventually, and if $\Phi$ is detected then $\Phi$ holds. If $\Phi$ is stable but is not locally stable, however, then the protocol is no longer complete: it may never be able to collect a consistent subcut and so never detect $\Phi$.

It is easy to extend our definition of locally stable properties to include more properties without sacrificing completeness or soundness. If we allow processes to continue to execute relevant events after $\Phi$ holds, but the number of relevant events that can be executed after $\Phi$ holds is finite, then $\Phi$ can always be detected by our protocol. This is because the processes will eventually reach a state in which no relevant events can be executed, thus allowing a consistent subcut to be collected. For 
example, consider a set of $n$ processes, each with its own variable $c_{i}$ that is initially zero and is repeatedly incremented. The formulation $\Phi:\left(\sum_{i=1}^{n} c_{i}\right) \geqq C$ for some constant $C$ is a stable property, and the relevant events are those that increase some $c_{i}$ that has a value less than $C$. In this case, each process can execute up to $C$ relevant events after the property holds and so we will call $\Phi$ locally stable. In general, if the number of relevant events that can be executed after $\Phi$ holds is bounded by $\lambda$, then the detection can take up to an additional $\lambda n$ token passes.

\section{Examples}

In this section, we instantiate the general protocol given above to obtain protocols for detecting specific properties. We first derive a known protocol for detecting termination. Termination is a simple property to formulate and detect. Its detection requires a full consistent cut, and a minimum of $n$ token passes. We then derive protocols for detecting two varieties of deadlock. The first, $k$-out-of- $m$ deadlock, is a general formulation of the deadlock problem that allows processes to make requests of a group of other processes. $k$-out-of- $m$ deadlock is not locally stable by our basic definition, but it is locally stable by the extension discussed at the end of Sect. 3.2. Finally, we derive a protocol for the simpler and more common formulation of deadlock in which processes can make requests of only one other process at a time. We call this form of deadlock $R P C$ deadlock.

\subsection{Termination}

We now instantiate the general protocol given above to obtain a protocol that detects termination in a distributed system. There are many variations of this property; the earliest that we know of is due to Dijkstra and Scholten [7]. The following definition is the same as that given in [12].

All processes are either active or idle. Only active processes can send messages. An active process may become idle at any time, and an idle process can become active only upon receipt of a message. The system is terminated when all processes in the system are idle and there are no messages in transit.

The local state of a process relative to termination consists of whether the process is active or idle and whether there is a message on an incoming channel. Therefore, the events that are relevant to termination are sending a message, receiving a message when idle, becoming idle, and becoming active. Each process will update its (weak) vector clock upon executing any of these events. Note that for this problem, we do not need to keep track of the contents of the messages exchanged between processes; only the number of messages is important. To capture the channel states, we have each process keep track of how many messages it has sent and received on each adjacent channel. The combined information of all of the processes will then yield the number of messages in transit on each channel: if $p_{i}$ has sent more messages to $p_{j}$ than $p_{j}$ has received from $p_{i}$, then there is at least one message on channel $C_{i, j}{ }^{6}$

We instantiate the general protocol given in Sect. 3.2 as follows.

Each process $p_{i}$ maintains the following local state variables:

- active ${ }_{i}$ Boolean $=$ true if and only if $p_{i}$ is active.

- $\operatorname{send}_{i}[1 \ldots n]$ : Integer array. $\operatorname{send}_{i}[j]=$ the number of messages that $p_{i}$ has sent to $p_{j}$. All are initially 0 .

- recvi $[1 \ldots n]$ : Integer array. $\operatorname{rec}_{i}[j]=$ the number of messages that $p_{i}$ has received from $p_{j}$. All are initially 0 .

When $p_{i}$ sends a message to $p_{j}, \operatorname{send}_{i}[j]$ is incremented. When $p_{i}$ receives a message from $p_{j}, \operatorname{recv}_{i}[j]$ is incremented. When $p_{i}$ becomes active or idle, active $i_{i}$ is set appropriately.

At some point, an idle process will start the detection protocol by circulating a token as described in Sect. 3.2. The termination condition can only be evaluated over a total global state (as opposed to a consistent proper subset of the process states), so a positive determination can be made only by the process $p_{f}$ that is the last to add its state to the token.

Process $p_{f}$ detects termination if and only if the following three conditions hold:

1. The timestamps in the token form a consistent cut;

2. All processes are idle: $\forall i$ : $_{\text {active }_{i}}=$ false;

3. There are no messages in transit: $\forall i, j: \operatorname{send}_{i}[j]=$ $\operatorname{recv}_{j}[i]$.

The following theorem and corollary show that Item 1 is redundant. The theorem assumes for simplicity that the buffered states are not collected in a token; the corollary removes this assumption.

Theorem 1. Let $\mathscr{B}=\left\{B_{i}: i=1,2, \ldots n\right\}$ be a a set of buffered state values that were recorded in a system that does not collect states in a token $D$. If in $\mathscr{B}, \forall i, j$ : $\operatorname{send}_{i}[j]=\operatorname{recv}_{j}[i]$, then the global state defined by $\mathscr{B}$ is consistent: $\forall i, j: B_{i} . V[i] \geqq B_{j} . V[i]$.

Proof. Suppose by way of contradiction that item 3 holds over $\mathscr{B}$ but the timestamps in $\mathscr{B}$ form an inconsistent cut: $\exists i, j: B_{i} . V[i]<B_{j} . V[i] . B_{j}, V[i]$ is advanced only when $p_{j}$ receives a message. Therefore, in order for $B_{j} . V[i]$ to advance beyond the recorded $B_{i} . V[i]$, there must have been a chain of messages between $p_{i}$ and $p_{j}$ between the time that $B_{i}$ was collected and the time that $B_{j}$ was collected. This implies that there is some $k$ such that the recorded $\operatorname{send}_{i}[k]<\operatorname{rec}_{k}[i]$, contradicting the assumption that item 3 holds.

Corollary 2. Let $\mathscr{B}=\left\{B_{i}: i=1,2, \ldots n\right\}$ be a a set of buffered state values that were collected in a token $D$. If in $D, \forall i, j: \operatorname{send}_{i}[j]=\operatorname{recv}_{j}[i]$, then the global state defined by $D$ is consistent: $\forall i, j: D_{i} . V[i] \geqq D_{j} . V[i]$.

\footnotetext{
${ }^{6}$ The counters used to store the number of messages sent and received by a process can become arbitrarily large. However, we will be using the counters in this protocol only to test whether channels are clear. For our purposes, if the number of messages that can be in transit between two processes is bounded by $k$ and the counters have a range of $[0 \ldots k]$, then the counters can safely wrap
} 
Proof. None of the events executed in collecting the buffered states into a token are relevant. Hence, collecting the states in this way has no effect on their consistency with respect to termination. The buffered states will therefore be consistent with respect to termination when items 2 and 3 hold.

Corollary 2 implies that the vector clocks need not be maintained. Furthermore, testing the states in the token for consistency can be done incrementally. For example, we can assign a total order to the processes and have the token passed along that total order. When process $p_{k}$ receives the token, it tests to see if

$$
\begin{aligned}
\neg \operatorname{active}_{k} \wedge(\forall \ell: 1 & \leqq \ell<k:\left(\operatorname{send}_{k}[l]\right. \\
& \left.\left.=\operatorname{recv}_{l}[k]\right) \wedge\left(\operatorname{send}_{l}[k]=\operatorname{recv}_{k}[l]\right)\right) .
\end{aligned}
$$

If this condition does not hold, then $p_{k}$ can drop the token. If the condition holds and $k=n$, then termination is detected; otherwise, $p_{k}$ fills in $D_{k}$ and passes the token to $p_{k+1}$.

This yields the protocol given in [10] as the channel counting protocol, which requires only $n$ messages to detect termination once it holds, and which can be further refined into a protocol that is space-efficient.

\section{$4.2 k$-out-of- $m$ deadlock}

We now instantiate the general protocol given in Sect. 3 to obtain a protocol that detects $k$-out-of- $m$ deadlock in a distributed system. This problem was first formulated and solved in [3]. In this formulation, a process can request $k$ resources from a pool of $m$ resources. We model the problem as follows:

A process is either active or blocked, where an active process is one that is not waiting for any other process. Active processes may issue $k$-out-of- $m$ requests in the following way. When an active process $p_{i}$ requires $k$ processes to carry out some request, it sends request messages to each of the $m$ processes that can perform this action. Process $p_{i}$ then becomes blocked, and waits until the action requested is carried out by at least $k$ of the $m$ processes. A process cannot send any further requests while blocked, but a process can receive request messages while blocked.

Only active processes can carry out a requested action. If a process $p_{j}$ receives a request while active, it will either become blocked by issuing a request to another process or carry out $p_{i}$ 's requested action within finite time. In the latter case, $p_{j}$ will send a grant message to $p_{i}$. When $p_{i}$ receives $k$ grant messages, it becomes active again. It then relinquishes the requests made to the rest of the processes to which it sent request messages by sending them relinquish messages. We assume that the recipient of a relinquish message acknowledges the message even if it is blocked and that the sender of a relinquish message waits for all acknowledgements before sending another request message. By doing so, we guarantee that $p_{i}$ can discard any grant messages received after the first $k$ are received.

The state of a process $p_{i}$ relative to $k$-out-of- $m$ deadlock consists of the number of grants needed for $p_{i}$ to become active and the current set of processes that $p_{i}$ is waiting for. We capture this state by having each process keep track of the processes on which it is blocked and the number of grant messages that it has sent and received on each adjacent channel.

We instantiate the general protocol given in Sec. 3.2 as follows. Each process $p_{i}$ maintains the following local state variables:

$-k_{i}$ : Integer $=$ the number of grant messages required for $p_{i}$ to become active (initially 0 ).

- $g_{-} \operatorname{send}_{i}[1 \ldots n]$ : Integer array. $g \operatorname{send}_{i}[j]$ is the number of grant messages that $p_{i}$ has sent to $p_{j}$ (all are initially 0 ).

$-g_{-} r e c v_{i}[1 \ldots n]$ : Integer array. $g \_r e c v_{i}[j]$ is the number of grant messages that $p_{i}$ has received from $p_{j}$ (all are initially 0 ).

$-w f_{i}$ : Integer set. These are the processes that $p_{i}$ is waiting for (initially empty). When $p_{i}$ sends a request message to $p_{j}, w f_{i}:=w f_{i} \cup\{j\}$; when $p_{i}$ receives a grant message from $p_{j}$ or sends a relinquish message to $p_{j}, w f_{i}:=w f_{i}-\{j\}$.

Deadlock is determined by constructing and reducing the system waits-for graph. This graph is constructed as follows:

- a waits-for edge is drawn from $p_{i}$ to $p_{j}$ if $\left.j \in w f_{i} \wedge\left(g_{-} \operatorname{send}_{j}[i]=g-\operatorname{recv}_{i}[j]\right)\right)$. That is, $p_{i}$ is waiting for a response from $p_{j}$ and no grant message is in transit from $p_{j}$ to $p_{i}$.

- the number of grants $\kappa_{i}$ required for $p_{i}$ to be unblocked is

$k_{i}-\sum_{j}\left(g_{-} \operatorname{send}_{j}[i]-g_{-} \operatorname{recv}_{i}[j]\right)$

That is, $\kappa_{i}$ is the number of grants that $p_{i}$ is waiting for less the number of grants in transit to $p_{i}$.

Deadlock is tested by reducing this graph as follows: if an edge points from $p_{i}$ to $p_{j}$ and $p_{j}$ is active, then the edge can be erased and $\kappa_{i}$ can be reduced by one; and if a process has $\kappa_{i}=0$, then all of its outgoing edges can be erased. The system is deadlocked if and only if there are edges that cannot be removed by following these two rules.

In this system, the relevant events of $p_{i}$ are those that change $k_{i}, g_{-} \operatorname{send}_{i}, g_{-} r e c v_{i}$, and $w f_{i}$. Hence, the relevant events are sending a request message, sending a grant message, receiving a grant message when blocked, and sending a relinquish message. We can now argue that $k$-out-of- $m$ deadlock is locally stable: a deadlocked process can execute only a bounded number of relevant events (namely, it can receive up to $k_{i}-1$ grant messages), and any valid global state that contains the local states of the deadlocked processes yields an irreducible waits-for graph.

The deadlock detection protocol is as follows. When a process $p_{i}$ wishes to test for deadlock, $p_{i}$ generates a token, fills $D_{i}$ with $\left(\left(k_{i}, g_{-} \operatorname{send}_{i}, g_{-} r e c v_{i}, w f_{i}\right), B_{i} . V[i]\right)$, and forwards the token to some $p_{j}: j \in w f_{i}$. When $p_{i}$ receives such a token, it fills $D_{i}$ with $\left(\left(k_{i}, g-s e n d_{i}\right.\right.$, $\left.\left.g_{\perp} r e c v_{i}, w f_{i}\right), B_{i} . V[i]\right)$ as before and then discards any collected values in the token that are not consistent with its state. If there exists a process $p_{j}$ whose state has not yet been collected $\left(D_{j}=\perp\right)$ and that may be blocking some process whose state has been collected $\left(\exists p_{k}\right.$ :

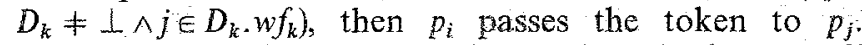
If there is no such process $p_{j}$, then $p_{i}$ then checks to see if 


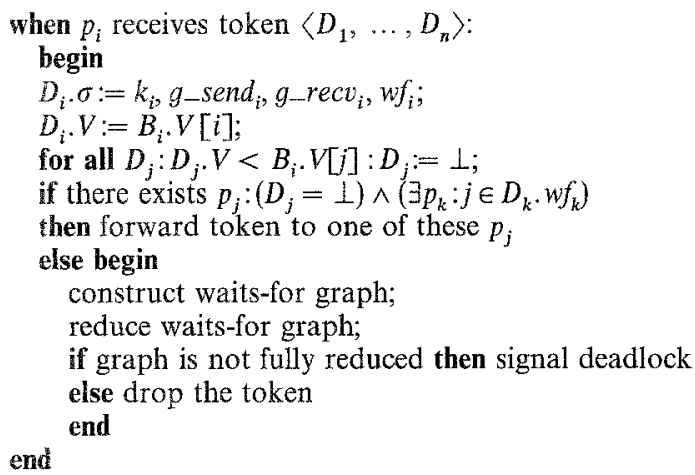

Fig. 7. Protocol for detecting $k$-out-of- $m$ deadlock

deadlock holds on the remaining values by constructing the waits-for graph and reducing it.

The full protocol is presented in Fig. 7. We assume that the process $p_{i}$ that generates the token does so because it suspects that it is involved in a deadlock; that is, $w f_{i}$ is not empty.

\subsection{RPC deadlock detection}

1-out-of-1 deadlock is a special case of $k$-out-of- $m$ deadlock that lends itself to further optimization. This type of deadlock is called RPC deadlock because it can occur in a remote procedure call system, where making a remote procedure call is analogous to requesting a resource from a single processor. The waits-for graph is constructed as for $k$-out-of- $m$ deadlock, except that $k_{i}=\left|w f_{i}\right|$ and thus need not be represented in the wait-for graph. Furthermore, relinquish messages are not needed and the waits-for graph is reducible if and only if it does not contain a cycle.

We can instantiate our protocol for detecting RPC deadlock as follows. As before, the relevant events are sending a request message (here, making an RPC request), sending a grant message (here, sending the reply to the RPC request), and receiving a grant message. Any blocked process $p_{i}$ can decide to detect deadlock by generating an empty token, inserting its buffered state into $D_{i}$, and passing the token to the (single) process in $w f_{i}$. When $p_{j}$ receives a token from $p_{i}, p_{j}$ checks whether it has sent a grant message to $p_{i}$ since $p_{i}$ sent the token. If $p_{j}$ has not done so and $w f_{j}$ is nonempty, $p_{j}$ passes the token on to the process in $w f_{j}$. A process detects deadlock when it receives a token that contains a complete cycle. The resulting protocol is shown in Fig. 8. If the waits-for graph contains a $d$-cycle, then the token need be passed only $d$ times. Note that since the token is passed along a path in the waits-for graph, the only part of process $p_{i}^{\prime} s$ state that needs to be recorded in the token is $g_{-} r e c v_{i}\left[w f_{i}\right]$.

This protocol can be further simplified by applying the following two theorems.

Theorem 3. Suppose $p_{i}$ receives a token from $p_{j}$ in the above protocol. If $\left(K . D_{j} . g_{-} r e c v_{j}[i]=g_{-}\right.$send $\left._{i}[j]\right)$ then $p_{j}$ has executed no relevant event since setting $K . D_{j}$.

Proof. Assume $\left(K . D_{j} . g-\operatorname{recv}_{j}[i]=g \_\operatorname{send}_{i}[j]\right)$. Since a process only forwards the token if it is blocked, the last

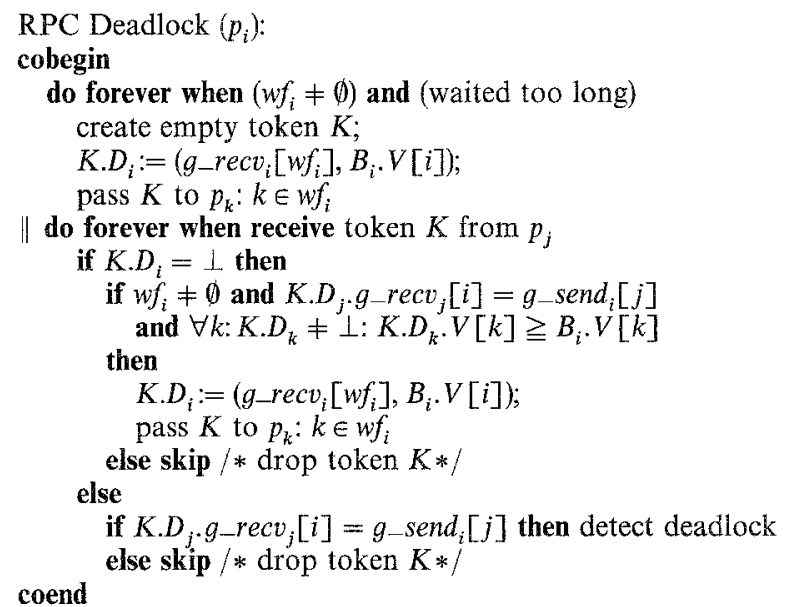

Fig. 8. RPC deadlock protocol, original

relevant event that $p_{j}$ can have executed before setting $K . D_{j}$ was to send a request message to $p_{i}$. The first relevant event that $p_{j}$ can have executed after setting $K . D_{j}$ is the receipt of a grant message from $p_{i}$. Since $\left(K . D_{j} . g \_r e c v_{j}[i]=g \_\right.$send $\left.d_{i}[j]\right), p_{i}$ has sent no grant messages to $p_{j}$ since $p_{j}$ sent the token to $p_{i}$. Hence, $p_{j}$ can have executed no relevant event since setting $K . D_{j}$.

Theorem 4. If $\left(K . D_{j} . g_{-} \operatorname{recv}_{j}[i]=g_{-} \operatorname{send}_{i}[j]\right)$ then no process that has set its value in $K$ has subsequently executed a relevant event.

Proof. Let $\ell$ be the number of values $D_{k}: K . D_{k} \neq \perp$, and assume that $\left(K \cdot D_{j} \cdot g \_r e c v_{j}[i]=g_{-} \operatorname{send}_{i}[j]\right)$. We will use induction on $\ell$.

Base case $(\ell=1)$. Follows directly from Theorem 3 .

Induction case $(\ell>1)$. By the induction hypothesis, no process prior to $p_{j}$ had executed a relevant event when $p_{j}$ received $K$. Since $K$ was transmitted to $p_{j}$ along a path in the waits-for graph, no process prior to $p_{j}$ can execute a relevant event until $p_{j}$ does, and by Theorem $3, p_{j}$ has not executed a relevant event since forwarding $K$ to $p_{i}$.

Theorem 4 implies

$$
\begin{aligned}
\left(g_{\operatorname{rec} v_{j}}[i]=g_{\left.-\operatorname{send}_{i}[j]\right) \Rightarrow}\right. & \left(\forall k: D_{k} \neq \perp: D_{k} . V\right. \\
& \left.=B_{k} \cdot V[k] \geqq B_{i} \cdot V[k]\right) .
\end{aligned}
$$

Thus, the vector clocks can be omitted and the token need only carry the identity of the process that initiated the test for deadlock. The resulting protocol, shown in Fig. 9, first appeared in [6].

The protocol in Fig. 8 can be easily generalized to detect and-deadlock ( $m$-out-of- $m$ requests), since a cycle in the waits-for graph is equivalent to deadlock in this case as well. The only change necessary is that when $p_{i}$ passes the token, it must replicate the token and pass a copy to each process in $w f_{i}$. With and-deadlock, a process can execute relevant events while deadlocked - a deadlocked process can receive a proper subset of its required grant messages. Thus, once deadlock holds among a set of $d$ processes, a total of $d *(n-d)$ relevant events can be executed by the deadlocked processes. Each such relevant event can lead to the token being dropped by one of the deadlocked 


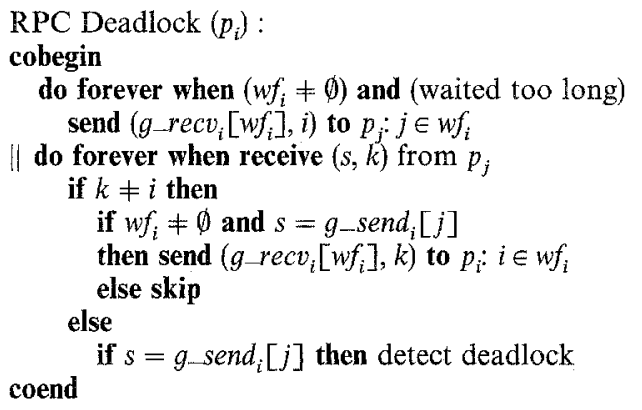

Fig. 9. RPC deadlock protocol, refined

processes. Therefore, the protocol may need to be restarted $O\left(n^{2}\right)$ times before deadlock is detected. On the other hand, if the protocol in Fig. 9 is run in parallel, then a token passed along a cycle will not be dropped. The resulting deadlock detection protocol is the one presented in [6].

\section{Conclusion}

This paper defined a proper subclass of the stable properties which we denote the locally stable properties. This subclass is interesting in that they are "terminationlike" or "deadlock-like": once a process is involved in establishing the stable property, the process will eventually cease changing its local state with respect to the stable property. Hence, in order to detect a locally stable property, a consistent cut need not be explicitly constructed - the relevant local states will form a consistent subcut implicitly. This leaves only the problem of detection.

In order to make this observation, we needed to define consistent cuts with respect to a global state predicate, and slightly extend the notion of vector clocks to accommodate our definition. We then gave a simple and decentralized protocol that detects when a locally stable property occurs in a distributed system. The protocol can be easily refined, which we illustrated by refining it to a known protocol for termination detection, a new protocol for $k$-out-of- $m$ deadlock detection, and a known protocol for RPC deadlock detection.

In the refinements to the two known protocols, the vector clocks proved redundant. This is because the representations of the channel states contained enough information to determine whether a set of local states constituted a consistent subcut. In fact, it is not hard to give sufficient conditions under which the vector clocks are redundant. Consider the following weaker definition of pairwise consistency: $e_{i}$ is pairwise consistent with $e_{j}$ if $\sigma_{j}$ does not reflect any messages sent by process $p_{i}$ through $e_{i}$ and $\sigma_{i}$ does not reflect any messages sent by process $p_{j}$ through $e_{j}$. Any set of events, one from each process in $P$, that are pairwise consistent according to this weaker definition still constitutes a consistent cut. This result can be generalized in order to determine whether a set of states is a consistent subcut.
One can test whether two states are (weakly) pairwise consistent by keeping track of the number of messages sent and received between the two processes. If all relevant send and receive events are counted, then it can be shown that these counters can be used in the place of weak vector clocks to test whether or not the set of states is a consistent global state. This was, in fact, the case in the protocol for termination detection. In our RPC deadlock detection protocol, only blocked processes forwarded the token, which allowed us to show that weak pairwise consistency among the states in the token implied that the subcut in the token was always consistent. The $k$-out-of- $m$ deadlock detection protocol, on the other hand, did not maintain counters for the relinquish messages, and so the vector clocks were not optimized away. Of course, the relinquish messages could have been counted, thereby eliminating the need for vector clocks, but doing so would have required $2 n$ counters per process rather than the $n$-valued vector clock.

We would like to determine what kinds of properties are locally stable. We know of two general classes: the locally stable properties of termination, global deadlock and some versions of distributed garbage detection can all be expressed as detecting no token in a generalized token passing system, yet deadlock of a subset of the processes does not seem to be so expressible. We are interested in whether there are other classes of locally stable properties.

Not all interesting stable properties are locally stable, however. For example, the property "the number of tokens is less than $k>0$ " in a token passing system that can lose but not regenerate tokens is stable. However, unless a process explicitly maintains in a local variable the number of tokens in the system (this could be done by some process periodically running a snapshot protocol), the property is not locally stable. We do not know if there are protocols that are more message-efficient than snapshot protocols for detecting such properties.

In Sect. 3.2 we extended the class of locally stable properties by allowing a process to execute a bounded number of relevant events after its state satisfies the stable property of interest. With this extension, all stable properties of a program are locally stable if all of the computations of that program contain only a bounded number of relevant events (for example, if the computations themselves have bounded lengths). This is because one could just wait for the computation to execute the bounded number of relevant events, collect the local states, and see if the property holds. Thus, the distinction of "locally stable" is interesting only for computations that contain an unbounded (finite or infinite) number of relevant events.

Our work was motivated by trying to derive messageefficient special-purpose detection protocols from a general detection protocol. In terms of our original goal, we have only been partially successful. Our protocol is most efficient when no process can execute a relevant event after the condition of interest holds. For example, in our discussion of the and-deadlock detection protocol in Sect. 4.3, we noted that our protocol may need to be restarted $O\left(n^{2}\right)$ times before detecting deadlock due to a deadlocked process being able to execute relevant receive events. Hence, we would like to better understand the notion of relevant 
events and weak vector clocks. An obvious approach would be to use a dynamic assignment of relevance to events rather than a static one. An event could then be denoted "relevant" based on what the process knows about the state of the system when it executes the event. This approach is suggestive of the derivation given in [5]. So far, however, we have not been able to derive a general and efficient detection protocol based on this generalization of relevant events: the most general way for a process to acquire knowledge of the state of the system beyond its own local state is to execute a snapshot protocol periodically. Doing so would extend the class of locally stable properties to all stable properties but also defeat the purpose of using a more efficient detection protocol. We know of no less-general method for dynamically assigning relevance to events that would lead to a class of stable properties that would be broader than locally stable and would have efficient detection protocols.

Finally, independently of this work, [15] has recently introduced the class of strong stable properties as a subset of stable properties. This class is very similar to the locally stable properties, although it is presented using different notation than ours. The general concrete protocol of [15] for detecting strong stable properties is essentially the same as ours when the set of relevant events is exactly the set of send and receive events. Furthermore, we know of stable properties that are locally stable but not strong stable. Hence, the set of strong stable properties appears to be a proper subset of the locally stable properties. Further investigation is needed to determine whether the class of locally stable properties is indeed weaker than the class of strong stable properties.

Acknowledgements. We would like to thank Özalp Babaoğlu, Gil Neiger, Fred Schneider, and Sam Toueg for their contributions to the ideas in this paper. We would also like to thank Ken Birman, Bard Bloom, Navin Budhiraja, Tushar Chandra, Mark Wood, and the anonymous referees for their valuable comments on earlier drafts of this paper.

\section{References}

1. Babaoğu Ö, Marzullo K: Consistent global states of distributed systems: fundamental concepts and mechanisms (chapter 4), pp 55-96. ACM, 1993. Edited by Sape Mullender

2. Birman K, Schiper A, Stephenson P: Lightweight causal and atomic group multicast. ACM Trans Comput Syst 9(3): 272-314 (1991)

3. Bracha $G$, Toueg S: A distributed algorithm for generalized deadlock detection. In: Proceedings of the Third Symposium on Principles of Distributed Computing, pp 285-301. ACM SIGPLAN/SIGOPS, August 1984

4. Chandy KM, Lamport L: Distributed snapshots: determining global states of distributed systems. ACM Trans Comput Syst 3(1): 63-75 (1985)

5. Chandy KM, Misra J: An example of stepwise refinement of distributed programs: quiescence detection. ACM Trans Program Lang Syst 8(3): 326-343 (1986)

6. Chandy KM, Misra J, Haas LM: Distributed deadlock detection. ACM Trans Comput Syst 1(2): 144-156 (1983)

7. Dijkstra EW, Scholten CS: Termination detection for diffusing computations. Inf Process Lett 11(1):1-4 (1980)

8. Holt RC: On deadlock in computer systems. $\mathrm{PhD}$ thesis, Cornell University, 1971

9. Lamport L: Time, clocks, and the ordering of events in a distributed system. Commun ACM 21(7): 558-565 (1978)

10. Mattern F: Algorithms for distributed termination detection. Distrib Comput 2(3):161-175 (1987)

11. Mattern F: Time and global states of distributed systems. In: M Cosnard et al. (eds) Proceedings of the International Workshop on Parallel and Distributed Algorithms. North-Holland 1989 , pp $215-226$

12. Misra J: Detecting termination of distributed computations using markers. In: Proceedings of the Second Symposium on Principles of Distributed Computing, pp 290-294. ACM SIGPLAN/SIGOPS, August 1983

13. Owicki S, Lamport L: Proving liveness properties of concurrent programs. ACM Trans Program Lang Syst 4(3): 455-495 (1982)

14. Peterson L L: Preserving context information in an IPC abstraction. In: Proceedings of the 6th Symposium on Reliability in Distributed Software and Database Systems, pp 22-31 (1987)

15. Schiper A, Sandoz A: Strong stable properties in distributed systems. Distrib Comput 8(3)

16. Schwarz R, Mattern F: Detecting causal relationships in distributed computations: in search for the holy grail. Distrib Comput 7(3): 149-174 (1994) 